\title{
The Halton sequence and its discrepancy in the Cantor expansion
}

\author{
Alena Haddley ${ }^{1}$ • Poj Lertchoosakul ${ }^{2}$. \\ Radhakrishnan Nair ${ }^{1}$
}

(C) The Author(s) 2016. This article is published with open access at Springerlink.com

\begin{abstract}
We consider variants of the Halton sequence in a generalized numeration system, called the Cantor expansion. We show that it provides a wealth of low-discrepancy sequences by giving an estimate of the (star) discrepancy of the Halton sequence in each bounded Cantor base. The techniques used in our estimation of the discrepancy are adapted from those developed by E.I. Atanassov.
\end{abstract}

Keywords Cantor expansion · Halton sequences · Hammersley point sets · Low-discrepancy sequences $\cdot$ Pseudorandom numbers $\cdot$ van der Corput sequences

Mathematics Subject Classification Primary 11J71 $\cdot 11 \mathrm{~K} 38 \cdot 11 \mathrm{~K} 45 \cdot$ Secondary 65C10

\section{Introduction}

Let $\omega=\left(x_{n}\right)_{n=1}^{\infty}$ be a sequence in $[0,1)^{S}$. A standard problem in numerical analysis is estimating the integral of a function, through a knowledge of its value at a finite number of points of the sequence. This is known as the Monte Carlo method in the case of stochastic sequences $\left(x_{n}\right)_{n=1}^{N}$ or the quasi-Monte Carlo method in the case of deterministic $\left(x_{n}\right)_{n=1}^{N}$. This is encapsulated in the famous Koksma-Hlawka inequality

$\bowtie$ Radhakrishnan Nair nair@liv.ac.uk

Alena Haddley

ajassova@liv.ac.uk

Poj Lertchoosakul

lertchoosakul@hotmail.com

1 Department of Mathematical Sciences, The University of Liverpool, Peach Street, Liverpool L69 7ZL, UK

2 Departamento de Matemática, Universidade de Brasília, Campus Darcy Ribeiro, L2 Norte, Asa Norte, Brasília-DF 70910-900, Brasil 


$$
\left|\int_{[0,1]^{s}} f(x) d x-\frac{1}{N} \sum_{n=1}^{N} f\left(x_{n}\right)\right| \leq V(f) D_{N}^{*}(\omega)
$$

for an arbitrary function $f$ on $[0,1]^{s}$ with bounded variation $V(f)$ in the sense of Hardy and Krause and any finite set of points $\left(x_{n}\right)_{n=1}^{N}$ with discrepancy

$$
D_{N}^{*}(\omega)=\sup _{J=\prod_{i=1}^{s}\left[0, z_{i}\right) \subseteq[0,1)^{s}}\left|\frac{A(J ; N ; \omega)}{N}-\lambda_{s}(J)\right| .
$$

Here $A(J ; N ; \omega)=\#\left\{1 \leq n \leq N: x_{n} \in J\right\}$ is the counting function, where $\lambda_{s}(J)$ denotes the $s$-dimensional Lebesgue measure of $J$ and the above supremum is taken over all rectangular solids $J=\prod_{i=1}^{s}\left[0, z_{i}\right)$ with $0<z_{i} \leq 1(1 \leq i \leq s)$. Note that $\lambda_{s}(J)=\prod_{i=1}^{s} z_{i}$. For more details on numerical integration, the reader can consult $[3,11]$ or [14]. Evidently, to estimate $\int_{[0,1]^{s}} f(x) d x$ sufficiently precisely, what is needed is a good bound for $D_{N}^{*}(\omega)$. The discrepancy is nothing other than a quantitative measure of uniformity of distribution. In particular, the sequence $\omega$ is uniformly distributed on $[0,1)^{s}$, if and only if $D_{N}^{*}(\omega) \rightarrow 0$ as $N \rightarrow \infty$. In a sense, the faster $D_{N}^{*}(\omega)$ decays as a function of $N$, the better uniformly distributed the sequence $\omega$ is. One of the fundamental obstructions in this subject is that there is a limit to how well distributed any sequence can be. This is encapsulated in the elementary inequality $D_{N}^{*}(\omega) \geq 1 / 2^{s} N(N \in \mathbb{N})$ whose proof makes an entertaining exercise. This opens the door to the deep subject of irregularities of distribution which addresses just what limitations there are to the uniformity of distribution of an arbitrary sequence, and the complementary problem of constructing sequences with discrepancy as small as possible. This latter issue is clearly central to the initial issue mentioned in this paper.

Perhaps the most famous example of a low-discrepancy sequence is the van der Corput sequence. In 1935, van der Corput [21] introduced a procedure to generate low-discrepancy sequences on $[0,1)$. These sequences are considered to be among the best distributed over $[0,1)$, and no other infinitely generated sequences can have discrepancy of smaller order of magnitude than van der Corput sequences. The technique of van der Corput is based on a very simple idea. Let $b>1$ be a natural number. Then every nonnegative integer $n$ has a $b$-adic representation of the form

$$
n=\sum_{j=1}^{\infty} n_{j} b^{j-1}=n_{1}+n_{2} b+n_{3} b^{2}+n_{4} b^{3}+\cdots,
$$

where $n_{j} \in\{0,1, \ldots, b-1\}(j \in \mathbb{N})$. The van der Corput sequence $\left(\phi_{b}(n)\right)_{n=0}^{\infty}$ in base $b$ is constructed by reversing the base $b$ representation of the sequence of nonnegative integers, where the radical-inverse function $\phi_{b}: \mathbb{N}_{0} \rightarrow[0,1)$ is defined by

$$
\phi_{b}\left(\sum_{j=1}^{\infty} n_{j} b^{j-1}\right)=\sum_{j=1}^{\infty} \frac{n_{j}}{b^{j}}=\frac{n_{1}}{b}+\frac{n_{2}}{b^{2}}+\frac{n_{3}}{b^{3}}+\cdots .
$$

In practical applications, a generalization of the van der Corput sequence to higher dimensions is more likely to be of practical use. In 1960, this was proposed by Halton [7]. Given coprime integers $b_{1}, \ldots, b_{s}$ all greater than 1 , the sequence $\left(\phi_{b_{1}}(n), \ldots, \phi_{b_{s}}(n)\right)_{n=0}^{\infty}$ is called the Halton sequence in bases $b_{1}, \ldots, b_{s}$.

It was known for a long time that the discrepancy of the first $N$ elements of the Halton sequence in bases $b_{1}, \ldots, b_{s}$ can be bounded by

$$
c\left(b_{1}, \ldots, b_{s}\right) \frac{(\log N)^{s}}{N}+O\left(\frac{(\log N)^{s-1}}{N}\right),
$$


for some constant $c\left(b_{1}, \ldots, b_{s}\right)>0$. For example, this was shown in $[4,6,7,15]$ and [18]. It is believed that the order $(\log N)^{s} / N$ is the best possible for an arbitrary infinite sequence. That this is the case when $s=1$ was proved by Schmidt [20]. For $s>1$, the question remains open. We shall call an infinite sequence $\omega$ in $[0,1)^{s}$ a low-discrepancy sequence if $D_{N}^{*}(\omega)=O\left((\log N)^{s} / N\right)$.

The question of how small the constant $c\left(b_{1}, \ldots, b_{s}\right)$ in (1.1) can be interesting from both a theoretical and a practical viewpoint. The articles referred to above show that this constant depends very strongly on the dimension $s$. The minimal value for this quantity can be obtained if we choose $b_{1}, \ldots, b_{s}$ to be the first $s$ prime numbers. But even in this case $c\left(b_{1}, \ldots, b_{s}\right)$ grows very fast to infinity if $s$ increases. This deficiency was overcome by Atanassov [1] who proved that

$$
c\left(b_{1}, \ldots, b_{s}\right)=\frac{1}{s !} \prod_{i=1}^{s} \frac{b_{i}-1}{\log b_{i}} .
$$

This estimate is so impressive that, when $b_{1}, \ldots, b_{s}$ are the first $s$ prime numbers, $c\left(b_{1}, \ldots, b_{s}\right) \rightarrow 0$ as $s \rightarrow \infty$.

In this paper, we introduce the Halton sequence in a generalized numeration system, which is induced by the $a$-adic integers and which is called the Cantor expansion, and give an estimate of its discrepancy by adapting the techniques developed by Atanassov. It is worth noting here that the van der Corput sequence and some one-dimensional low-discrepancy sequences with respect to the Cantor expansion were studied in [2] and [5]. Note also that it was mentioned in [8] about the Halton sequence in a more generalized numeration system than the Cantor expansion, called the $G$-expansion; however, the paper aimed to study the Halton sequence in some fixed non-integer bases and did not touch on the Halton sequence with respect to dynamical bases.

We now summarize the contents of this paper. In Sect. 2, we introduce the concept of a generalized numeral system. Then we define the Halton sequence induced by this generalized system and state our main result on the estimate of discrepancy of the Halton sequence. In Sects. 3 and 4, we collect all preliminary lemmas and prove our main result, respectively. In Sect. 5, we prove an estimate of discrepancy of the van der Corput sequence in a generalized numeration system without the restriction on boundedness of inducing sequences. Then we pose an open problem regarding an extension of our results. Finally, we introduce in Sect. 6 the Hammersley point set induced by the generalized numeration system and show that it provides a wealth of low-discrepancy point sets by giving an estimate of its discrepancy.

\section{A generalized Halton sequence}

Let $\underline{b}=\left(b_{j}\right)_{j=1}^{\infty}$ be a sequence of natural numbers greater than 1 . Then it is clear that every nonnegative integer $n$ has a unique $\underline{b}$-adic representation of the form

$$
n=\sum_{j=1}^{\infty} n_{j} b_{1} \cdots b_{j-1}=n_{1}+n_{2} b_{1}+n_{3} b_{1} b_{2}+n_{4} b_{1} b_{2} b_{3}+\cdots,
$$

where $n_{j} \in\left\{0,1, \ldots, b_{j}-1\right\}(j \in \mathbb{N})$. This $\underline{b}$-adic representation is also called the Cantor expansion of $n$ with respect to the Cantor base $\underline{b}$. Moreover, every real number $x \in[0,1)$ 
has a $\underline{b}$-adic expansion of the form

$$
x=\sum_{j=1}^{\infty} \frac{x_{j}}{b_{1} \cdots b_{j}}=\frac{x_{1}}{b_{1}}+\frac{x_{2}}{b_{1} b_{2}}+\frac{x_{3}}{b_{1} b_{2} b_{3}}+\cdots,
$$

where $x_{j} \in\left\{0,1, \ldots, b_{j}-1\right\}(j \in \mathbb{N})$. The $x_{j}$ can be calculated by the greedy algorithm

$$
x_{1}=\left[x b_{1}\right] \quad \text { and } \quad x_{j}=\left[\left\{x b_{1} \cdots b_{j-1}\right\} b_{j}\right],
$$

where $[\alpha]$ and $\{\alpha\}$ denote the integer part and the fractional part of $\alpha$, respectively. The idea of this generalized numeration system stems from the $a$-adic integers, which is a class of locally compact topological groups and possesses a symbolic dynamical structure. For more details on the $a$-adic integers, see [9, pp. 106-117].

Define the radical-inverse function $\phi_{\underline{b}}: \mathbb{N}_{0} \rightarrow[0,1)$ by

$$
\phi_{\underline{b}}\left(\sum_{j=1}^{\infty} n_{j} b_{1} \cdots b_{j-1}\right)=\sum_{j=1}^{\infty} \frac{n_{j}}{b_{1} \cdots b_{j}}=\frac{n_{1}}{b_{1}}+\frac{n_{2}}{b_{1} b_{2}}+\frac{n_{3}}{b_{1} b_{2} b_{3}}+\cdots .
$$

The van der Corput sequence in base $\underline{b}$ is defined as $\left(\phi_{\underline{b}}(n)\right)_{n=0}^{\infty}$. This sequence was studied in [2] and [5], where it was proved to be a low-discrepancy sequence with some restriction on the Cantor base $\underline{b}$. Furthermore, the sequence was shown, without any restriction on the Cantor base, to be uniformly distributed mod 1 in [13] and to be a low-discrepancy sequence in [12].

Let $\underline{b}_{1}=\left(b_{1, j}\right)_{j=1}^{\infty}, \ldots, \underline{b}_{s}=\left(b_{s, j}\right)_{j=1}^{\infty}$ be $s$ sequences of natural numbers greater than 1 such that, for all $j_{1}, j_{2} \in \mathbb{N}$ and all $1 \leq i_{1}<i_{2} \leq s$, we have $b_{i_{1}, j_{1}}$ and $b_{i_{2}, j_{2}}$ coprime. The Halton sequence in bases $\underline{b}_{1}, \ldots, \underline{b}_{s}$ is defined to be $\omega=\left(\phi_{\underline{b}_{1}}(n), \ldots, \phi_{\underline{b}_{s}}(n)\right)_{n=0}^{\infty}$.

The following is our main result and gives an estimate of the discrepancy of the Halton sequence in bases of bounded sequences.

Main Theorem 2.1 Let $\underline{b}_{1}=\left(b_{1, j}\right)_{j=1}^{\infty}, \ldots, \underline{b}_{s}=\left(b_{s, j}\right)_{j=1}^{\infty}$ be $s$ bounded sequences of natural numbers greater than 1 such that, for all $j_{1}, j_{2} \in \mathbb{N}$ and all $1 \leq i_{1}<i_{2} \leq s$, we have $b_{i_{1}, j_{1}}$ and $b_{i_{2}, j_{2}}$ coprime. Suppose that $\omega$ is the Halton sequence in bases $\underline{b}_{1}, \ldots, \underline{b}_{s}$. Then, for any $N \geq 1$, we have

$$
N D_{N}^{*}(\omega) \leq \frac{1}{s !} \prod_{i=1}^{s}\left(\frac{\left\lfloor M_{i} / 2\right\rfloor \log N}{\log m_{i}}+s\right)+\sum_{l=0}^{s-1} \frac{M_{l+1}}{l !} \prod_{i=1}^{l}\left(\frac{\left\lfloor M_{i} / 2\right\rfloor \log N}{\log m_{i}}+l\right),
$$

where $M_{i}=\max \left(b_{i, j}\right)_{j=1}^{\infty}$ and $m_{i}=\min \left(b_{i, j}\right)_{j=1}^{\infty}(1 \leq i \leq s)$.

Corollary 2.2 Let $\underline{b}_{1}=\left(b_{1, j}\right)_{j=1}^{\infty}, \ldots, \underline{b}_{s}=\left(b_{s, j}\right)_{j=1}^{\infty}$ be $s$ bounded sequences of natural numbers greater than 1 such that, for all $j_{1}, j_{2} \in \mathbb{N}$ and all $1 \leq i_{1}<i_{2} \leq s$, we have $b_{i_{1}, j_{1}}$ and $b_{i_{2}, j_{2}}$ coprime. Suppose that $\omega$ is the Halton sequence in bases $\underline{b}_{1}, \ldots, \underline{b}_{s}$. Then, for any $N \geq 1$, we have

$$
D_{N}^{*}(\omega) \leq c\left(\underline{b}_{1}, \ldots, \underline{b}_{s}\right) \frac{(\log N)^{s}}{N}+O\left(\frac{(\log N)^{s-1}}{N}\right)
$$

with

$$
c\left(\underline{b}_{1}, \ldots, \underline{b}_{s}\right)=\frac{1}{s !} \prod_{i=1}^{s} \frac{\left\lfloor M_{i} / 2\right\rfloor}{\log m_{i}},
$$

where $M_{i}=\max \left(b_{i, j}\right)_{j=1}^{\infty}$ and $m_{i}=\min \left(b_{i, j}\right)_{j=1}^{\infty}(1 \leq i \leq s)$.

This shows that the Halton sequence is a low-discrepancy sequence. 


\section{Preliminary lemmas}

In order to prove Theorem 2.1, we need the following five lemmas. These preliminary results are adapted from [3] whose ideas go back to Atanassov [1].

Lemma 3.1 Let $\underline{b}_{1}=\left(b_{1, j}\right)_{j=1}^{\infty}, \ldots, \underline{b}_{s}=\left(b_{s, j}\right)_{j=1}^{\infty}$ be $s$ arbitrary sequences of natural numbers greater than 1 such that, for all $j_{1}, j_{2} \in \mathbb{N}$ and all $1 \leq i_{1}<i_{2} \leq s$, we have $b_{i_{1}, j_{1}}$ and $b_{i_{2}, j_{2}}$ coprime. Suppose that $\omega$ is the Halton sequence in bases $\underline{b}_{1}, \ldots, \underline{b}_{s}$. Let $J$ be an interval of the form

$$
J=\prod_{i=1}^{s}\left[\frac{u_{i}}{b_{i, 1} \cdots b_{i, k_{i}}}, \frac{v_{i}}{b_{i, 1} \cdots b_{i, k_{i}}}\right)
$$

with integers $0 \leq u_{i}<v_{i} \leq b_{i, 1} \cdots b_{i, k_{i}}$ and $k_{i} \geq 1$ for all $1 \leq i \leq s$. Then the inequality

$$
\left|A(J ; N ; \omega)-N \lambda_{s}(J)\right| \leq \prod_{i=1}^{s}\left(v_{i}-u_{i}\right)
$$

holds for every $N \in \mathbb{N}$. Moreover, for every $N \leq \prod_{i=1}^{s} b_{i, 1} \cdots b_{i, k_{i}}$, we have $A(J ; N ; \omega) \leq$ $\prod_{i=1}^{s}\left(v_{i}-u_{i}\right)$.

Proof For each $n \in \mathbb{N}_{0}$, we denote the $\underline{b}_{i}$-adic expansion of $n$ by

$$
n=n_{1}^{(i)}+n_{2}^{(i)} b_{i, 1}+n_{3}^{(i)} b_{i, 1} b_{i, 2}+n_{4}^{(i)} b_{i, 1} b_{i, 2} b_{i, 3}+\cdots,
$$

where $n_{j}^{(i)} \in\left\{0,1, \ldots, b_{i, j}-1\right\}(j \in \mathbb{N})$. Choose $\ell=\left(l_{1}, \ldots, l_{s}\right) \in \mathbb{N}_{0}^{s}$ such that, for all $1 \leq i \leq s$, we have $0 \leq l_{i}<b_{i, 1} \cdots b_{i, k_{i}}$ with the expansion

$$
l_{i}=l_{i, k_{i}}+l_{i, k_{i}-1} b_{i, k_{i}}+l_{i, k_{i}-2} b_{i, k_{i}} b_{i, k_{i}-1}+\cdots+l_{i, 1} b_{i, k_{i}} \cdots b_{i, 2},
$$

where $l_{i, k_{i}-j} \in\left\{0,1, \ldots, b_{i, k_{i}-j}-1\right\}\left(0 \leq j \leq k_{i}-1\right)$. We consider the interval

$$
J_{\ell}=\prod_{i=1}^{s}\left[\frac{l_{i}}{b_{i, 1} \cdots b_{i, k_{i}}}, \frac{l_{i}+1}{b_{i, 1} \cdots b_{i, k_{i}}}\right) .
$$

Then the $n$th element $\omega_{n}$ of the Halton sequence is contained in $J_{\ell}$, if and only if, for all $1 \leq i \leq s$,

$$
\frac{l_{i, 1}}{b_{i, 1}}+\cdots+\frac{l_{i, k_{i}}}{b_{i, 1} \cdots b_{i, k_{i}}} \leq \frac{n_{1}^{(i)}}{b_{i, 1}}+\frac{n_{2}^{(i)}}{b_{i, 1} b_{i, 2}}+\cdots<\frac{l_{i, 1}}{b_{i, 1}}+\cdots+\frac{l_{i, k_{i}}}{b_{i, 1} \cdots b_{i, k_{i}}}+\frac{1}{b_{i, 1} \cdots b_{i, k_{i}}} .
$$

This is however equivalent to $n_{1}^{(i)}=l_{i, 1}, \ldots, n_{k_{i}}^{(i)}=l_{i, k_{i}}$ which in turn is equivalent to $n \equiv l_{i, 1}+l_{i, 2} b_{i, 1}+\cdots+l_{i, k_{i}} b_{i, 1} \cdots b_{i, k_{i}-1}\left(\bmod b_{i, 1} \cdots b_{i, k_{i}}\right)$ for all $1 \leq i \leq s$.

As $b_{1, j_{1}}, \ldots, b_{s, j_{s}}$ are pairwise coprime for all $\left(j_{1}, \ldots, j_{s}\right) \in \mathbb{N}^{s}$, we obtain from the Chinese Remainder Theorem that every $\prod_{i=1}^{s} b_{i, 1} \cdots b_{i, k_{i}}$ consecutive elements of the Halton sequence, contain exactly one element in $J_{\ell}$ or, in other words, $A\left(J_{\ell} ; t \prod_{i=1}^{s} b_{i, 1} \cdots b_{i, k_{i}} ; \omega\right)=$ $t$ for all $t \in \mathbb{N}$, and hence

$$
A\left(J_{\ell} ; t \prod_{i=1}^{s} b_{i, 1} \cdots b_{i, k_{i}} ; \omega\right)-\left(t \prod_{i=1}^{s} b_{i, 1} \cdots b_{i, k_{i}}\right) \lambda_{s}\left(J_{\ell}\right)=0 .
$$

Therefore, for every $N \in \mathbb{N}$, we obtain

$$
\left|A\left(J_{\ell} ; N ; \omega\right)-N \lambda_{s}\left(J_{\ell}\right)\right| \leq 1 .
$$


Now we write the interval $J$ as a disjoint union of intervals of the form $J_{\ell}$,

$$
J=\bigcup_{l_{1}=u_{1}}^{v_{1}-1} \cdots \bigcup_{l_{s}=u_{s}}^{v_{s}-1} J_{\ell}
$$

where $\ell=\left(l_{1}, \ldots, l_{s}\right)$. We then have

$$
\left|A(J ; N ; \omega)-N \lambda_{s}(J)\right| \leq \sum_{l_{1}=u_{1}}^{v_{1}-1} \ldots \sum_{l_{s}=u_{s}}^{v_{s}-1}\left|A\left(J_{\ell} ; N ; \omega\right)-N \lambda_{s}\left(J_{\ell}\right)\right| \leq \prod_{i=1}^{s}\left(v_{i}-u_{i}\right),
$$

which proves the first assertion.

For every $N \leq \prod_{i=1}^{s} b_{i, 1} \cdots b_{i, k_{i}}$, we always have $A\left(J_{\ell} ; N ; \omega\right) \leq 1$ for each $\ell=$ $\left(l_{1}, \ldots, l_{s}\right) \in \mathbb{N}_{0}^{S}$ with $0 \leq l_{i}<b_{i, 1} \cdots b_{i, k_{i}}$ for all $1 \leq i \leq s$, and hence

$$
A(J ; N ; \omega)=\sum_{l_{1}=u_{1}}^{v_{1}-1} \cdots \sum_{l_{s}=u_{s}}^{v_{s}-1} A\left(J_{\ell} ; N ; \omega\right) \leq \prod_{i=1}^{s}\left(v_{i}-u_{i}\right) .
$$

This was the second assertion of the lemma.

Lemma 3.2 Let $\underline{b}_{1}=\left(b_{1, j}\right)_{j=1}^{\infty}, \ldots, \underline{b}_{s}=\left(b_{s, j}\right)_{j=1}^{\infty}$ be $s$ arbitrary sequences of natural numbers greater than 1 . For each $N \in \mathbb{N}$, let $d\left(\underline{b}_{1}, \ldots, \underline{b}_{s} ; N\right)$ be the number of tuples $\left(k_{1}, \ldots, k_{s}\right) \in \mathbb{N}^{s}$ such that $\prod_{i=1}^{s} b_{i, 1} \cdots b_{i, k_{i}} \leq N$. Then we have

$$
d\left(\underline{b}_{1}, \ldots, \underline{b}_{s} ; N\right) \leq \frac{1}{s !} \prod_{i=1}^{s} \frac{\log N}{\log m_{i}},
$$

where $m_{i}=\min \left(b_{i, j}\right)_{j=1}^{\infty}(1 \leq i \leq s)$.

Proof Let $N \in \mathbb{N}$. Assume that $k=\left(k_{1}, \ldots, k_{s}\right) \in \mathbb{N}^{s}$ satisfies $\prod_{i=1}^{s} b_{i, 1} \cdots b_{i, k_{i}} \leq N$. Notice that we immediately have $m_{1}^{k_{1}} \cdots m_{s}^{k_{s}} \leq N$. Then the interval $E_{k}=\prod_{i=1}^{s}\left[k_{i}-1, k_{i}\right)$ of volume 1 is entirely contained in the simplex

$$
S=\left\{\left(x_{1}, \ldots, x_{s}\right) \in[0, \infty)^{s}: x_{1} \log m_{1}+\cdots+x_{s} \log m_{s} \leq \log N\right\}
$$

of volume $\frac{1}{s !} \prod_{i=1}^{s} \frac{\log N}{\log m_{i}}$. Hence, we obtain

$$
d\left(\underline{b}_{1}, \ldots, \underline{b}_{s} ; N\right)=\lambda_{s}\left(\bigcup_{E_{k} \subseteq S} E_{k}\right) \leq \lambda_{s}(S)=\frac{1}{s !} \prod_{i=1}^{s} \frac{\log N}{\log m_{i}},
$$

as required.

Lemma 3.3 Let $\underline{b}_{1}=\left(b_{1, j}\right)_{j=1}^{\infty}, \ldots, \underline{b}_{s}=\left(b_{s, j}\right)_{j=1}^{\infty}$ be $s$ arbitrary sequences of natural numbers greater than 1 . Suppose that $\left(z_{k}^{(1)}\right)_{k=0}^{\infty}, \ldots,\left(z_{k}^{(s)}\right)_{k=0}^{\infty}$ are $s$ bounded sequences of nonnegative real numbers such that $z_{0}^{(i)} \leq 1$ and $z_{k}^{(i)} \leq f_{i}$ for all $k \in \mathbb{N}$ and all $1 \leq i \leq s$. Then, for any $N \geq 1$, we have

$$
\sum_{\substack{\left(k_{1}, \ldots, k_{s}\right) \in \mathbb{N}_{0}^{s} \\ \prod_{i=1}^{s} b_{i, 1} \cdots b_{i, k_{i}} \leq N}} \prod_{i=1}^{s} z_{k_{i}}^{(i)} \leq \frac{1}{s !} \prod_{i=1}^{s}\left(f_{i} \frac{\log N}{\log m_{i}}+s\right),
$$

where $m_{i}=\min \left(b_{i, j}\right)_{j=1}^{\infty}(1 \leq i \leq s)$. 
Proof Let $\mathfrak{u} \subseteq\{1, \ldots, s\}$. By Lemma 3.2, the number of $s$-tuples $\left(k_{1}, \ldots, k_{s}\right)$ such that $k_{i}>0$ if $i \in \mathfrak{u}, k_{i}=0$ if $i \notin \mathfrak{u}$, and $\prod_{i \in \mathfrak{u}} b_{i, 1} \cdots b_{i, k_{i}} \leq N$ is bounded above by $\frac{1}{|\mathfrak{u}| !} \prod_{i \in \mathfrak{u}} \frac{\log N}{\log m_{i}}$. Moreover, each of these $s$-tuples contributes at most $\prod_{i \in \mathfrak{u}} f_{i}$ to the sum on the left hand side in the statement of the lemma. From this, invoking the inequality $\frac{1}{|\mathfrak{u}| !} \leq \frac{s^{s-|\mathfrak{u}|}}{s !}$, we obtain

$$
\begin{aligned}
\sum_{\substack{\left(k_{1}, \ldots, k_{s}\right) \in \mathbb{N}_{0}^{s} \\
\prod_{i=1}^{s} b_{i, 1} \cdots b_{i, k_{i}} \leq N}} \prod_{i=1}^{s} z_{k_{i}}^{(i)} & \leq \sum_{\mathfrak{u} \subseteq\{1, \ldots, s\}} \frac{1}{|\mathfrak{u}| !} \prod_{i \in \mathfrak{u}} f_{i} \frac{\log N}{\log m_{i}} \\
& \leq \frac{1}{s !} \sum_{\mathfrak{u} \subseteq\{1, \ldots, s\}} s^{s-|\mathfrak{u}|} \prod_{i \in \mathfrak{u}} f_{i} \frac{\log N}{\log m_{i}} \\
& =\frac{1}{s !} \prod_{i=1}^{s}\left(f_{i} \frac{\log N}{\log m_{i}}+s\right),
\end{aligned}
$$

and this is the desired result.

Now we need to introduce some notation. Let $J \subseteq \mathbb{R}^{s}$ be an interval. Then a signed splitting of $J$ is a collection of not necessarily disjoint intervals $J_{1}, \ldots, J_{r}$ together with signs $\varepsilon_{1}, \ldots, \varepsilon_{r} \in\{-1,1\}$ such that, for all $x \in J$, we have

$$
\sum_{\substack{i=1 \\ x \in J_{i}}}^{r} \varepsilon_{i}=1
$$

A function $v$ on the class of intervals in $\mathbb{R}^{s}$ is said to be additive if, for each pair of disjoint intervals $A, B$ in $\mathbb{R}^{s}$, we have $v(A \cup B)=v(A)+v(B)$. It is not hard to see that the $s$ dimensional Lebesgue measure $\lambda_{s}$ and the counting function $A(\cdot ; N ; \omega)$ are the examples we are particularly interested in. It is not hard to check that, for any additive function $v$ on the class of intervals in $\mathbb{R}^{s}$, we have

$$
v(J)=\sum_{i=1}^{r} \varepsilon_{i} v\left(J_{i}\right),
$$

where $\left(J_{1}, \ldots, J_{r} ; \varepsilon_{1}, \ldots, \varepsilon_{r}\right)$ is a signed splitting of $J$, and here we use $v\left(J_{i}\right)=v\left(J_{i} \cap J\right)$ ( $1 \leq i \leq r)$. The following result is taken directly from [1] (see also [3] for a detailed proof).

Lemma 3.4 ([1, Lemma 3.5]) Let $J=\prod_{i=1}^{s}\left[0, z_{i}\right)$ be an $s$-dimensional box. For each $1 \leq i \leq s$, let $\left(z_{k, i}\right)_{k=1, \ldots, n_{i}}$ be an arbitrary finite sequence of numbers in $[0,1]$. Define further $z_{0, i}=0$ and $z_{n_{i}+1, i}=z_{i}$ for all $1 \leq i \leq s$. Then the collection of intervals

$$
\prod_{i=1}^{s}\left[\min \left(z_{k_{i}, i}, z_{k_{i}+1, i}\right), \max \left(z_{k_{i}, i}, z_{k_{i}+1, i}\right)\right)
$$

together with the signs $\varepsilon_{k_{1}, \ldots, k_{s}}=\prod_{i=1}^{s} \operatorname{sgn}\left(z_{k_{i}+1, i}-z_{k_{i}, i}\right)$ for $0 \leq k_{i} \leq n_{i}$ and $1 \leq i \leq s$ define a signed splitting of the interval $J$.

For the proof of Theorem 2.1, we need a digit expansion of reals $z \in[0,1)$ in $\left(b_{j}\right)_{j=1}^{\infty}$-adic base which uses signed digits. The next lemma shows that such an expansion exists. 
Lemma 3.5 Let $\underline{b}=\left(b_{j}\right)_{j=1}^{\infty}$ be an arbitrary sequence of natural numbers greater than 1 . Then every $z \in[0,1)$ can be written in the form

$$
z=a_{0}+\frac{a_{1}}{b_{1}}+\frac{a_{2}}{b_{1} b_{2}}+\frac{a_{3}}{b_{1} b_{2} b_{3}}+\cdots
$$

with integer digits $a_{0}, a_{1}, a_{2}, \ldots$ such that $a_{0} \in\{0,1\}$ and $-\left\lfloor\frac{b_{j}-1}{2}\right\rfloor \leq a_{j} \leq\left\lfloor\frac{b_{j}}{2}\right\rfloor$ for all $j \in \mathbb{N}$. This expansion is called a signed $\underline{b}$-adic expansion of $z$.

Proof For each $j \in \mathbb{N}$, let $c_{j}=\left\lfloor\frac{b_{j}-1}{2}\right\rfloor$. Define

$$
x=\frac{c_{1}}{b_{1}}+\frac{c_{2}}{b_{1} b_{2}}+\frac{c_{3}}{b_{1} b_{2} b_{3}}+\cdots \in[0,1) .
$$

For $z \in[0,1)$, we have $z+x \in[0,2)$ with $\underline{b}$-adic expansion

$$
z+x=u_{0}+\frac{u_{1}}{b_{1}}+\frac{u_{2}}{b_{1} b_{2}}+\frac{u_{3}}{b_{1} b_{2} b_{3}}+\cdots,
$$

where $u_{0} \in\{0,1\}$, and where $u_{j} \in\left\{0,1, \ldots, b_{j}-1\right\}(j \in \mathbb{N})$. Therefore,

$$
z=u_{0}+\frac{u_{1}-c_{1}}{b_{1}}+\frac{u_{2}-c_{2}}{b_{1} b_{2}}+\frac{u_{3}-c_{3}}{b_{1} b_{2} b_{3}}+\cdots
$$

with $-\left\lfloor\frac{b_{j}-1}{2}\right\rfloor \leq u_{j}-c_{j} \leq b_{j}-1-\left\lfloor\frac{b_{j}-1}{2}\right\rfloor=\left\lfloor\frac{b_{j}}{2}\right\rfloor$ for all $j \in \mathbb{N}$, as required.

\section{Proof of the main theorem}

Proof of Theorem 2.1 Let $J=\prod_{i=1}^{s}\left[0, z_{i}\right) \subseteq[0,1)^{s}$. According to Lemma 3.5, for all $1 \leq i \leq s$, we consider the signed $\underline{b}_{i}$-adic expansion of $z_{i}$ of the form

$$
z_{i}=a_{i, 0}+\frac{a_{i, 1}}{b_{i, 1}}+\frac{a_{i, 2}}{b_{i, 1} b_{i, 2}}+\frac{a_{i, 3}}{b_{i, 1} b_{i, 2} b_{i, 3}}+\cdots
$$

with $a_{i, 0} \in\{0,1\}$ and $-\left\lfloor\frac{b_{i, j}-1}{2}\right\rfloor \leq a_{i, j} \leq\left\lfloor\frac{b_{i, j}}{2}\right\rfloor(j \in \mathbb{N})$.

For all $1 \leq i \leq s$, let $n_{i}=\left\lfloor\frac{\log N}{\log m_{i}}\right\rfloor+1$, and for $1 \leq k \leq n_{i}$, define the truncations of the expansions

$$
z_{k, i}=a_{i, 0}+\frac{a_{i, 1}}{b_{i, 1}}+\frac{a_{i, 2}}{b_{i, 1} b_{i, 2}}+\cdots+\frac{a_{i, k-1}}{b_{i, 1} \cdots b_{i, k-1}},
$$

and let $z_{0, i}=0$ and $z_{n_{i}+1, i}=z_{i}$.

According to Lemma 3.4, the collection of intervals

$$
J_{k_{1}, \ldots, k_{s}}=\prod_{i=1}^{s}\left[\min \left(z_{k_{i}, i}, z_{k_{i}+1, i}\right), \max \left(z_{k_{i}, i}, z_{k_{i}+1, i}\right)\right)
$$

together with the signs $\varepsilon_{k_{1}, \ldots, k_{s}}=\prod_{i=1}^{s} \operatorname{sgn}\left(z_{k_{i}+1, i}-z_{k_{i}, i}\right)$ for $0 \leq k_{i} \leq n_{i}$ and $1 \leq i \leq s$ defines a signed splitting of the interval $J$.

Since both $\lambda_{s}$ and $A(\cdot ; N ; \omega)$ are additive functions on the set of intervals, we obtain that

$$
\begin{aligned}
A(J ; N ; \omega)-N \lambda_{s}(J) & =\sum_{k_{1}=0}^{n_{1}} \cdots \sum_{k_{s}=0}^{n_{s}} \varepsilon_{k_{1}, \ldots, k_{s}}\left(A\left(J_{k_{1}, \ldots, k_{s}} ; N ; \omega\right)-N \lambda_{s}\left(J_{k_{1}, \ldots, k_{s}}\right)\right) \\
& =\Sigma_{1}+\Sigma_{2},
\end{aligned}
$$


where $\Sigma_{1}$ denotes the sum over all $\left(k_{1}, \ldots, k_{s}\right)$ such that $\prod_{i=1}^{s} b_{i, 1} \cdots b_{i, k_{i}} \leq N$ and $\Sigma_{2}$ denotes the remaining part of the above sum.

First we deal with the sum $\Sigma_{1}$. For any $1 \leq i \leq s$, the length of the interval $\left[\min \left(z_{k_{i}, i}, z_{k_{i}+1, i}\right), \max \left(z_{k_{i}, i}, z_{k_{i}+1, i}\right)\right)$ is $\left|a_{i, k_{i}} / b_{i, 1} \cdots b_{i, k_{i}}\right|$, and also the limit points of this interval are rationals with denominator $b_{i, 1} \cdots b_{i, k_{i}}$. It is worth keeping in mind that, due to the choice of $n_{i}$, we must have $k_{i}<n_{i}$ when $\prod_{i=1}^{s} b_{i, 1} \cdots b_{i, k_{i}} \leq N$. Accordingly, the intervals $J_{k_{1}, \ldots, k_{s}}$ are of the form as considered in Lemma 3.1 from which we obtain

$$
\left|A\left(J_{k_{1}, \ldots, k_{s}} ; N ; \omega\right)-N \lambda_{s}\left(J_{k_{1}, \ldots, k_{s}}\right)\right| \leq \prod_{i=1}^{s}\left|a_{i, k_{i}}\right|
$$

We have $\left|a_{i, k_{i}}\right| \leq\left\lfloor\frac{b_{i, k_{i}}}{2}\right\rfloor \leq\left\lfloor\frac{M_{i}}{2}\right\rfloor=: f_{i}$. An application of Lemma 3.3 yields that

$$
\Sigma_{1} \leq \frac{1}{s !} \prod_{i=1}^{s}\left(\frac{\left\lfloor M_{i} / 2\right\rfloor \log N}{\log m_{i}}+s\right)
$$

It remains to estimate $\Sigma_{2}$. To this end, we split the set of $s$-tuples $\left(k_{1}, \ldots, k_{s}\right)$ for which $\prod_{i=1}^{s} b_{i, 1} \cdots b_{i, k_{i}}>N$ into disjoint sets $B_{0}, B_{1}, \ldots, B_{s-1}$ where we set $B_{0}=$ $\left\{\left(k_{1}, \ldots, k_{s}\right) \in \mathbb{N}_{0}^{s}: b_{1,1} \cdots b_{1, k_{1}}>N\right\}$ and, for $1 \leq l \leq s-1$,

$$
B_{l}=\left\{\left(k_{1}, \ldots, k_{s}\right) \in \mathbb{N}_{0}^{s}: \prod_{i=1}^{l} b_{i, 1} \cdots b_{i, k_{i}} \leq N \text { and } \prod_{i=1}^{l+1} b_{i, 1} \cdots b_{i, k_{i}}>N\right\} .
$$

For a fixed $1 \leq l \leq s-1$ and a fixed $l$-tuple $\left(k_{1}, \ldots, k_{l}\right)$ with $\prod_{i=1}^{l} b_{i, 1} \cdots b_{i, k_{i}} \leq N$, define $r$ to be the largest integer such that

$$
\left(\prod_{i=1}^{l} b_{i, 1} \cdots b_{i, k_{i}}\right)\left(b_{l+1,1} \cdots b_{l+1, r-1}\right) \leq N
$$

It follows that the tuple $\left(k_{1}, \ldots, k_{l}, k_{l+1}, \ldots, k_{s}\right)$ is contained in $B_{l}$, if and only if $k_{l+1} \geq r$.

Therefore, for any $0 \leq l \leq s-1$ and fixed $k_{1}, \ldots, k_{l} \in \mathbb{N}_{0}$ such that $\prod_{i=1}^{l} b_{i, 1} \cdots b_{i, k_{i}} \leq$ $N$, we have that

$$
\sum_{\substack{k_{l+1}, \ldots, k_{s} \in \mathbb{N} \\\left(k_{1}, \ldots, k_{s}\right) \in B_{l}}} \varepsilon_{k_{1}, \ldots, k_{s}}\left(A\left(J_{k_{1}, \ldots, k_{s}} ; N ; \omega\right)-N \lambda_{s}\left(J_{k_{1}, \ldots, k_{s}}\right)\right)= \pm\left(A(L ; N ; \omega)-N \lambda_{s}(L)\right),
$$

where

$$
\begin{aligned}
L= & \prod_{i=1}^{l}\left[\min \left(z_{k_{i}, i}, z_{k_{i}+1, i}\right), \max \left(z_{k_{i}, i}, z_{k_{i}+1, i}\right)\right) \\
& \times\left[\min \left(z_{r, l+1}, z_{l+1}\right), \max \left(z_{r, l+1}, z_{l+1}\right)\right) \times \prod_{i=l+2}^{s}\left[0, z_{i}\right) .
\end{aligned}
$$


Let $\left(k_{1}, \ldots, k_{s}\right) \in B_{l}$. As

$$
\begin{aligned}
\left|z_{l+1}-z_{r, l+1}\right| & =\left|\frac{a_{l+1, r}}{b_{l+1,1} \cdots b_{l+1, r}}+\frac{a_{l+1, r+1}}{b_{l+1,1} \cdots b_{l+1, r+1}}+\frac{a_{l+1, r+2}}{b_{l+1,1} \cdots b_{l+1, r+2}}+\cdots\right| \\
& =\frac{1}{b_{l+1,1} \cdots b_{l+1, r-1}}\left|\frac{a_{l+1, r}}{b_{l+1, r}}+\frac{a_{l+1, r+1}}{b_{l+1, r} b_{l+1, r+1}}+\frac{a_{l+1, r+2}}{b_{l+1, r} b_{l+1, r+1} b_{l+1, r+2}}+\cdots\right| \\
& \leq \frac{1}{b_{l+1,1} \cdots b_{l+1, r-1}}\left(\frac{\left.\left\lfloor b_{l+1, r}\right\rfloor\right\rfloor}{b_{l+1, r}}+\frac{\left\lfloor b_{l+1, r+1}\right\rfloor}{b_{l+1, r} b_{l+1, r+1}}+\frac{\left\lfloor b_{l+1, r+2}\right\rfloor}{b_{l+1, r} b_{l+1, r+1} b_{l+1, r+2}}+\cdots\right) \\
& \leq \frac{1}{b_{l+1,1} \cdots b_{l+1, r-1}}\left(\frac{1}{2}+\frac{1}{2 b_{l+1, r}}+\frac{1}{2 b_{l+1, r} b_{l+1, r+1}}+\cdots\right) \\
& \leq \frac{1}{b_{l+1,1} \cdots b_{l+1, r-1}}\left(\frac{1}{2}+\frac{1}{2^{2}}+\frac{1}{2^{3}}+\cdots\right) \\
& =\frac{1}{b_{l+1,1} \cdots b_{l+1, r-1}},
\end{aligned}
$$

it follows that the interval $\left[\min \left(z_{r, l+1}, z_{l+1}\right), \max \left(z_{r, l+1}, z_{l+1}\right)\right)$ is contained in some interval

$$
\left[\frac{u}{b_{l+1,1} \cdots b_{l+1, r}}, \frac{v}{b_{l+1,1} \cdots b_{l+1, r}}\right)
$$

for $u, v \in \mathbb{N}_{0}$ with $v-u \leq b_{l+1, r}$. Hence, $L$ is contained in the interval

$$
\begin{aligned}
L^{\prime}= & \prod_{i=1}^{l}\left[\min \left(z_{k_{i}, i}, z_{k_{i}+1, i}\right), \max \left(z_{k_{i}, i}, z_{k_{i}+1, i}\right)\right) \\
& \times\left[\frac{u}{b_{l+1,1} \cdots b_{l+1, r}}, \frac{v}{b_{l+1,1} \cdots b_{l+1, r}}\right) \times[0,1)^{s-l-1} .
\end{aligned}
$$

Since $\left(k_{1}, \ldots, k_{s}\right) \in B_{l}$, we have $N<\left(\prod_{i=1}^{l} b_{i, 1} \cdots b_{i, k_{i}}\right)\left(b_{l+1,1} \cdots b_{l+1, r}\right)$ and $N \geq$ $\prod_{i=1}^{l} b_{i, 1} \cdots b_{i, k_{i}}$. The latter fact implies that $k_{i}<n_{i}$ for all $1 \leq i \leq s$. Thus, an application of Lemma 3.1 yields that

$$
A(L ; N ; \omega) \leq A\left(L^{\prime} ; N ; \omega\right) \leq b_{l+1, r} \prod_{i=1}^{l}\left|a_{i, k_{i}}\right| .
$$

But on the other hand, we also have that $N \lambda_{s}(L) \leq b_{l+1, r} \prod_{i=1}^{l}\left|a_{i, k_{i}}\right|$. Hence,

$$
\left|A(L ; N ; \omega)-N \lambda_{s}(L)\right| \leq b_{l+1, r} \prod_{i=1}^{l}\left|a_{i, k_{i}}\right| \leq M_{l+1} \prod_{i=1}^{l} c_{i, k_{i}},
$$

where $c_{i, k_{i}}=1$ if $k_{i}=0$ and $c_{i, k_{i}}=\left\lfloor\frac{M_{i}}{2}\right\rfloor$ otherwise.

Summing up, we obtain

$$
\begin{aligned}
\left|\Sigma_{2}\right| & \leq \sum_{l=0}^{s-1} \sum_{\substack{\prod_{1}, \ldots, k_{l} \in \mathbb{N}_{0} \\
\prod_{i=1}^{l} b_{i, 1} \cdots b_{i, k_{i}} \leq N}}\left|\sum_{\left(k_{1}, \ldots, k_{s}\right) \in B_{l}} \epsilon_{k_{1}, \ldots, k_{s}}\left(A\left(J_{k_{1}, \ldots, k_{s}} ; N ; \omega\right)-N \lambda_{s}\left(J_{k_{1}, \ldots, k_{s}}\right)\right)\right| \\
& \leq \sum_{l=0}^{s-1} \sum_{\substack{k_{1}, \ldots, k_{l} \in \mathbb{N}_{0} \\
\prod_{i=1}^{l} b_{i, 1} \cdots b_{i, k_{i}} \leq N}} M_{l+1} \prod_{i=1}^{l} c_{i, k_{i}} \leq \sum_{l=0}^{s-1} \frac{M_{l+1}}{l !} \prod_{i=1}^{l}\left(\frac{\left\lfloor M_{i} / 2\right\rfloor \log N}{\log m_{i}}+l\right),
\end{aligned}
$$

where we have used Lemma 3.3 again. Hence, the result follows. 


\section{An open problem}

We have seen that the Halton sequence in bases of bounded sequences $\underline{b}_{1}, \ldots, \underline{b}_{s}$ is a low-discrepancy sequence. It is of theoretical interest to ask whether the assumption of boundedness of the base sequences $\underline{b}_{1}, \ldots, \underline{b}_{s}$ can be removed or not.

The following statement shows that we can remove the restriction on the boundedness when $s=1$. That is, the van der Corput sequence in base $\underline{b}=\left(b_{j}\right)_{j=1}^{\infty}$ of an arbitrary sequence of natural numbers greater than 1 is a low-discrepancy sequence. Note that the proof is developed from the classical dyadic case in [10, pp. 127-128].

Proposition 5.1 Let $\underline{b}=\left(b_{j}\right)_{j=1}^{\infty}$ be an arbitrary sequence of natural numbers greater than 1. Suppose that $\omega$ is the van der Corput sequence in base $\underline{b}$. Then, for any $N \in \mathbb{N}$, we have

$$
N D_{N}^{*}(\omega) \leq \frac{\log N}{\log m}+1,
$$

where $m=\min \left(b_{j}\right)_{j=1}^{\infty}$.

Note that Proposition 5.1 gives a better estimation of discrepancy than the case $s=1$ of Theorem 2.1 and also that of [2, Théorème 4.5]. To prove this result, we need to introduce a notation and two preliminary lemmas.

A finite sequence $0 \leq c_{1}<c_{2}<\cdots<c_{L}$ of points from the interval $[0,1)$ is called an arithmetic progression with difference $\eta$ if $c_{i+1}-c_{i}=\eta$ for all $1 \leq i \leq L-1$.

The first lemma gives an estimation of discrepancy of an arithmetic progressions.

Lemma 5.2 ([16, Theorem 2.1]) Let $c_{1}<c_{2}<\cdots<c_{L}$ be an arithmetic progression with difference $\eta$. Then we have

$$
D_{L}^{*}\left(c_{1}, \ldots, c_{L}\right) \leq \min (\eta, 1 / L)
$$

The next simple lemma is useful for estimating the discrepancy of a sequence which can be decomposed into a number of subsequences with small discrepancy.

Lemma 5.3 ([18, Theorem 2.6, Ch. 2]) For $1 \leq k \leq K$, let $\omega_{k}$ be a sequence of $N_{k}$ elements in $[0,1)$ with discrepancy $D_{N_{k}}^{*}\left(\omega_{k}\right)$. Let $\omega$ be a superposition of $\omega_{1}, \ldots, \omega_{K}$, that is, a sequence obtained by listing in some order the terms of $\omega_{k}$. We set $N=N_{1}+\cdots+N_{K}$, which will be the number of elements of $\omega$. Then we have

$$
N D_{N}^{*}(\omega) \leq \sum_{k=1}^{K} N_{k} D_{N_{k}}^{*}\left(\omega_{k}\right) .
$$

Proof of Proposition 5.1 We can always represent a given $N \in \mathbb{N}$ by its $\underline{b}$-adic expansion

$$
N=N_{1}+N_{2} b_{1}+N_{3} b_{1} b_{2}+\cdots+N_{k} b_{1} \cdots b_{k-1},
$$

where $k \in \mathbb{N}$ and $N_{j} \in\left\{0,1, \ldots, b_{j}-1\right\}(1 \leq j \leq k)$. Partition the interval $[0, N]$ of integers into $k$ subintervals $I_{1}, \ldots, I_{k}$ as follows. First, put $I_{1}=\left[0, N_{k} b_{1} \cdots b_{k-1}\right]$. Then, for each $1<j \leq k$, we define $I_{j}$ as the interval

$$
\left[N_{k} b_{1} \cdots b_{k-1}+\cdots+N_{k-j+2} b_{1} \cdots b_{k-j+1}+1, N_{k} b_{1} \cdots b_{k-1}+\cdots+N_{k-j+1} b_{1} \cdots b_{k-j}\right] \text {. }
$$

Note that the proof idea of splitting up the range of $0,1, \ldots, N$ in this way is due to Niederreiter [17]. 
An integer $n \in I_{j}(1 \leq j \leq k)$ can be written in the form

$$
n=N_{k} b_{1} \cdots b_{k-1}+\cdots+N_{k-j+2} b_{1} \cdots b_{k-j+1}+1+\sum_{i=1}^{k-j+1} n_{i} b_{0} \cdots b_{i-1},
$$

where $b_{0}=1$ and $n_{i} \in\left\{0,1, \ldots, b_{i}-1\right\}(1 \leq i \leq k-j+1)$ such that $n_{k-j+1}<N_{k-j+1}$. In fact, we get all $N_{k-j+1} b_{1} \cdots b_{k-j}$ integers in $I_{j}$ if we let $n_{i}$ run through all possible combinations. It now follows that

$$
\begin{aligned}
\phi_{\underline{b}}(n) & =\frac{N_{k}}{b_{1} \cdots b_{k}}+\cdots+\frac{N_{k-j+2}}{b_{1} \cdots b_{k-j+2}}+\frac{1}{b_{1} \cdots b_{k-j+1}}+\sum_{i=1}^{k-j+1} \frac{n_{i}}{b_{1} \cdots b_{i}} \\
& =x_{j}+\sum_{i=1}^{k-j+1} \frac{n_{i}}{b_{1} \cdots b_{i}},
\end{aligned}
$$

where $x_{j}$ only depends on $j$, and not on $n$. If $n$ runs through $I_{j}$, then $\sum_{i=1}^{k-j+1} \frac{n_{i}}{b_{1} \cdots b_{i}}$ runs through all fractions $0, \frac{1}{b_{1}}, \ldots, \frac{b_{1}-1}{b_{1}}, \frac{1}{b_{1} b_{2}}, \ldots, \sum_{i=1}^{k-j} \frac{b_{i}-1}{b_{1} \cdots b_{i}}+\frac{N_{k-j+1}}{b_{1} \cdots b_{k-j+1}}$ in some order. Moreover, we note that

$$
0 \leq x_{j}=\frac{N_{k}}{b_{1} \cdots b_{k}}+\cdots+\frac{N_{k-j+2}}{b_{1} \cdots b_{k-j+2}} \leq \frac{b_{k} \cdots b_{k-j+2}-1}{b_{1} \cdots b_{k}} \leq \frac{1}{b_{1} \cdots b_{k-j+1}} .
$$

We deduce that if the $\phi_{b}(n)\left(n \in I_{j}\right)$ are ordered according to their magnitude, then we obtain a sequence $\omega_{j}$ of $\bar{N}_{k-j+1} b_{1} \cdots b_{k-j}$ elements that is a true arithmetic progression with parameters $\eta_{j}=\frac{1}{b_{1} \cdots b_{k-j+1}}$. It now follows immediately from Lemma 5.2 that the discrepancy of each $\omega_{j}$, multiplied by the number of elements in $\omega_{j}$, is at most 1 . Combining this with Lemma 5.3 and the fact that $\phi_{\underline{b}}(0), \phi_{\underline{b}}(1), \ldots, \phi_{\underline{b}}(N)$ is decomposed into $k$ sequences $\omega_{j}$, we obtain $N D_{N}^{*}(\omega) \leq k$.

It remains to estimate $k$ in terms of $N$. By (5.1), we have $N \geq b_{1} \cdots b_{k-1} \geq m^{k-1}$, and so we obtain $k \leq(\log N / \log m)+1$. This completes the proof of Proposition 5.1.

In general, it is likely to be true that the Halton sequence in arbitrary bases of sequences is a low-discrepancy sequence.

Problem 5.4 Let $s>1$, and let $\underline{b}_{1}=\left(b_{1, j}\right)_{j=1}^{\infty}, \ldots, \underline{b}_{s}=\left(b_{s, j}\right)_{j=1}^{\infty}$ be $s$ arbitrary sequences of natural numbers greater than 1 such that, for all $j_{1}, j_{2} \in \mathbb{N}$ and all $1 \leq i_{1}<i_{2} \leq s$, we have $b_{i_{1}, j_{1}}$ and $b_{i_{2}, j_{2}}$ coprime. Suppose that $\omega$ is the Halton sequence in bases $\underline{b}_{1}, \ldots, \underline{b}_{s}$. Then we have

$$
D_{N}^{*}(\omega) \leq c\left(\underline{b}_{1}, \ldots, \underline{b}_{s}\right) \frac{(\log N)^{s}}{N}+O\left(\frac{(\log N)^{s-1}}{N}\right),
$$

where $c\left(\underline{b}_{1}, \ldots, \underline{b}_{s}\right)>0$ is a constant.

If the conjecture is true, then it is natural to ask further whether the constant $c\left(\underline{b}_{1}, \ldots, \underline{b}_{s}\right)$ can be reduced to a similar form to that in Corollary 2.2.

\section{A generalized Hammersley point set}

Based on the $(s-1)$-dimensional Halton sequence, we can introduce a finite $s$-dimensional point set which is known as the Hammersley point set. 
Let $\underline{b}_{1}=\left(b_{1, j}\right)_{j=1}^{\infty}, \ldots, \underline{b}_{s-1}=\left(b_{s-1, j}\right)_{j=1}^{\infty}$ be $s-1$ sequences of natural numbers greater than 1 such that, for all $j_{1}, j_{2} \in \mathbb{N}$ and all $1 \leq i_{1}<i_{2} \leq s-1$, we have $b_{i_{1}, j_{1}}$ and $b_{i_{2}, j_{2}}$ coprime. The Hammersley point set in bases $\underline{b}_{1}, \ldots, \underline{b}_{s-1}$ consisting of $N$ points in $[0,1)^{s}$ is defined to be the point set

$$
\mathcal{P}=\left\{\left(\frac{n}{N}, \phi_{\underline{b}_{1}}(n), \ldots, \phi_{\underline{b}_{s-1}}(n)\right): 0 \leq n \leq N-1\right\} .
$$

We deduce a discrepancy bound for the Hammersley point set with the help of Theorem 2.1 in combination with the following general result from [18] that goes back to Roth [19].

Lemma 6.1 ([18, Lemma 3.7]) Let $\omega=\left(x_{n}\right)_{n=0}^{\infty}$ be an arbitrary sequence in $[0,1)^{s-1}$ with discrepancy $D_{N}^{*}(\omega)$. For $N \in \mathbb{N}$, let $\mathcal{P}$ be the point set consisting of $\left(n / N, x_{n}\right)$ in $[0,1)^{s}$ for $n=0,1, \ldots, N-1$. Then we have

$$
N D_{N}^{*}(\mathcal{P}) \leq \max _{1 \leq N^{\prime} \leq N} N^{\prime} D_{N^{\prime}}^{*}(\omega)+1 .
$$

Theorem 6.2 Let $\underline{b}_{1}=\left(b_{1, j}\right)_{j=1}^{\infty}, \ldots, \underline{b}_{s-1}=\left(b_{s-1, j}\right)_{j=1}^{\infty}$ be $s-1$ arbitrary sequences of natural numbers greater than 1 such that, for all $j_{1}, j_{2} \in \mathbb{N}$ and all $1 \leq i_{1}<i_{2} \leq s-1$, we have $b_{i_{1}, j_{1}}$ and $b_{i_{2}, j_{2}}$ are coprime. For $N \in \mathbb{N}$, let $\mathcal{P}$ be the Hammersley point set in bases $\underline{b}_{1}, \ldots, \underline{b}_{s-1}$ consisting of $N$ points. Then, for any $N \geq 2$, we have

$N D_{N}^{*}(\mathcal{P}) \leq \frac{1}{(s-1) !} \prod_{i=1}^{s-1}\left(\frac{\left\lfloor M_{i} / 2\right\rfloor \log N}{\log m_{i}}+s-1\right)+\sum_{l=0}^{s-2} \frac{M_{l+1}}{l !} \prod_{i=1}^{l}\left(\frac{\left\lfloor M_{i} / 2\right\rfloor \log N}{\log m_{i}}+l\right)+1$, where $M_{i}=\max \left\{b_{i, j} \in \underline{b}_{i}: b_{i, 1} \cdots b_{i, j} \leq N\right\}$ and $m_{i}=\min \left\{b_{i, j} \in \underline{b}_{i}: b_{i, 1} \cdots b_{i, j} \leq N\right\}$ $(1 \leq i \leq s-1)$.

Corollary 6.3 Let $\underline{b}_{1}=\left(b_{1, j}\right)_{j=1}^{\infty}, \ldots, \underline{b}_{s-1}=\left(b_{s-1, j}\right)_{j=1}^{\infty}$ be $s-1$ bounded sequences of natural numbers greater than 1 such that, for all $j_{1}, j_{2} \in \mathbb{N}$ and all $1 \leq i_{1}<i_{2} \leq s-1$, we have $b_{i_{1}, j_{1}}$ and $b_{i_{2}, j_{2}}$ coprime. For $N \in \mathbb{N}$, let $\mathcal{P}$ be the Hammersley point set in bases $\underline{b}_{1}, \ldots, \underline{b}_{s-1}$ consisting of $N$ points. Then, for any $N \geq 2$, we have

$$
D_{N}^{*}(\mathcal{P}) \leq c\left(\underline{b}_{1}, \ldots, \underline{b}_{s-1}\right) \frac{(\log N)^{s-1}}{N}+O\left(\frac{(\log N)^{s-2}}{N}\right)
$$

with

$$
c\left(\underline{b}_{1}, \ldots, \underline{b}_{s-1}\right)=\frac{1}{(s-1) !} \prod_{i=1}^{s-1} \frac{\left\lfloor M_{i} / 2\right\rfloor}{\log m_{i}},
$$

where $M_{i}=\max \left(b_{i, j}\right)_{j=1}^{\infty}$ and $m_{i}=\min \left(b_{i, j}\right)_{j=1}^{\infty}(1 \leq i \leq s)$.

A point set $\mathcal{P}$ consisting of $N$ points in $[0,1)^{S}$ is called a low-discrepancy point set if $D_{N}^{*}(\mathcal{P})=O\left((\log N)^{s-1} / N\right)$. In this sense, the generalized Hammersley point set is a low-discrepancy point set.

Acknowledgements We wish to thank the referee for his/her careful and valuable comments, which were very helpful in improving the presentation of this manuscript. The research of Poj Lertchoosakul was partially supported by the Grant 346300 for IM PAN from the Simons Foundation.

Open Access This article is distributed under the terms of the Creative Commons Attribution 4.0 International License (http://creativecommons.org/licenses/by/4.0/), which permits unrestricted use, distribution, and reproduction in any medium, provided you give appropriate credit to the original author(s) and the source, provide a link to the Creative Commons license, and indicate if changes were made. 


\section{References}

1. E.I. Atanassov, On the discrepancy of the Halton sequences. Math. Balkanica (N.S.) 18, 15-32 (2004)

2. H. Chaix, H. Faure, Discrépance et diaphonie en dimension un. Acta Arith. 63, 103-141 (1993)

3. J. Dick, F. Pillichshammer, Digital Nets and Sequences: Discrepancy Theory and Quasi-Monte Carlo Integration (Cambridge University Press, Cambridge, 2010)

4. H. Faure, Suites à faible discrépance dans $T^{s}$ (Publ. Dép. Math, Université de Limoges, France, 1980)

5. H. Faure, Discrépances de suites associées à un système de numération (en dimension un). Bull. Soc. Math. France 109, 143-182 (1981)

6. H. Faure, Discrépance de suites associées à un systéme de numéracion (en dimension s). Acta Arith. 41, 337-351 (1982)

7. J.H. Halton, On the efficiency of certain quasi-random sequences of points in evaluating multi-dimensional integrals. Numer. Math. 2, 84-90 (1960)

8. M. Hofer, M.R. Iacò, R. Tichy, Ergodic properties of $\beta$-adic Halton sequences. Ergod. Theory Dynam. Syst. 35, 895-909 (2015)

9. E. Hewitt, K.A. Ross, Abstract Harmonic Analysis I: Structure of Topological Groups, Integration Theory and Group Representations, Grundlehren der Mathematischen Wissenschaften, vol. 115 (Springer, New York, 1979)

10. L. Kuipers and H. Niederreiter, Uniform Distribution of Sequences, Pure and Applied Mathematics (WileyInterscience (John Wiley \& Sons), New York, 1974)

11. C. Lemieux, Monte Carlo and Quasi-Monte Carlo Sampling. Springer Series in Statistics (Springer, New York, 2009)

12. P. Lertchoosakul, On the Metric Theory of Numbers in Non-Achimedean Settings, Ph.D. Thesis, University of Liverpool, UK, 2014

13. P. Lertchoosakul, R. Nair, Distribution functions for subsequences of the van der Corput sequence. Indag. Math. (N.S.) 24, 593-601 (2013)

14. G. Leobacher, F. Pillichshammer, Introduction to Quasi-Monte Carlo Integration and Applications, Compact Textbook in Mathematics, Birkhäuser/Springer, Cham, 2014

15. H.G. Meijer, The discrepancy of a $g$-adic sequence. Indag. Math. 30, 54-66 (1968)

16. H. Niederreiter, Almost-arithmetic progressions and uniform distribution. Trans. Am. Math. Soc. 161, 283-292 (1971)

17. H. Niederreiter, Point sets and sequences with small discrepancy. Monatsh. Math. 104, 273-337 (1987)

18. H. Niederreiter, Random Number Generation and Quasi-Monte Carlo Methods, CBMS-NSF Regional Conference Series in Applied Mathematics, 63, Society for Industrial and Applied Mathematics (SIAM), Philadelphia, PA, 1992

19. K.F. Roth, On irregularities of distribution. Mathematika 1, 73-79 (1954)

20. W.M. Schmidt, Irregularities of distribution. VII. Acta Arith. 21, 45-50 (1972)

21. J.G. van der Corput, Verteilungsfunktionen. Proc. Ned. Akad. v. Wet. 38, 813-821 (1935) 